\title{
Antihelium-3 fluxes near Earth using data-driven estimates for annihilation cross section
}

3 Laura Šerkšnyte் ${ }^{a, *}$ on behalf of the ALICE Collaboration

4 (a complete list of authors can be found at the end of the proceedings)

$5 \quad{ }^{a}$ The Technical University of Munich,

$6 \quad$ James-Franck-Str. 1, Garching, Germany

$7 \quad$ E-mail: laura.serksnyte@tum.de

Antinuclei found in cosmic rays could provide a smoking gun signal for dark matter as this signal is virtually background free. The study of ${ }^{3} \overline{\mathrm{He}}$ cosmic rays requires the knowledge of their production, propagation in the galaxy and annihilation cross-section. While the former two have been already estimated with data-driven methods, there were no experimental data available for

8 the ${ }^{3} \overline{\mathrm{He}}$ inelastic cross section. We measured for the first time the inelastic cross section of ${ }^{3} \overline{\mathrm{He}}$ using the ALICE detector itself as a target. To study the effect of ${ }^{3} \overline{\mathrm{He}}$ annihilation in the galaxy and estimate the transparency of the galaxy, the ${ }^{3} \overline{\mathrm{He}}$ source functions and annihilation cross sections were implemented in GALPROP.

$37^{\text {th }}$ International Cosmic Ray Conference (ICRC 2021)

July 12 th $-23 r d, 2021$

Online - Berlin, Germany

\footnotetext{
${ }^{*}$ Presenter
} 


\section{Introduction}

The yet undetected dark matter is expected to account for the missing mass in the Universe. Weakly interacting massive particles (WIMPs) are believed to annihilate and produce particleantiparticle pairs of the ordinary matter. Antinuclei produced in our galaxy by dark matter annihilation processes would propagate and be detected as antimatter cosmic rays in the Earth's proximity. However, antinuclei can also be produced by cosmic ray collisions with the interstellar gas. This contribution represents a background source for dark matter searches. Luckily, the cosmic ray flux from the two sources peaks at different kinetic energies and the dark matter signal is virtually background free. The measurement of antinuclei cosmic rays would hence provide a smoking gun signal for dark matter.

The modelling of the behaviour of the background and signal is mandatory to draw conclusions for antinuclei fluxes. This work focuses on the description of the behaviour of ${ }^{3} \overline{\mathrm{He}}$ cosmic rays in our galaxy. The full-scale description of the fluxes can be implemented in a transport equation which requires the knowledge of three main components: the production cross section of antinuclei, their annihilation cross section and their propagation in the galaxy. The transport equation can be solved numerically using the publicly available GALPROP code [1].

The ${ }^{3} \overline{\mathrm{He}}$ production cross sections and the propagation parameters can be constrained using existing experimental data, while no measurement of the inelastic cross section of the ${ }^{3} \overline{\mathrm{He}}$ was available up to now. We provide the first ever measurement of the inelastic cross section of ${ }^{3} \overline{\mathrm{He}}$ using the ALICE detector as a target material. We implement the ${ }^{3} \overline{\mathrm{He}}$ cosmic ray source functions and our obtained inelastic cross sections in GALPROP to study the effect of annihilation processes in the Galaxy.

\section{2. ${ }^{3} \overline{\mathrm{He}}$ Inelastic Cross Section Measurement}

At LHC energies, matter and antimatter are produced in almost equal amounts. The ${ }^{3} \mathrm{He}$ and ${ }^{3} \overline{\mathrm{He}}$ are produced rather copiously as it can be seen in Fig. 1, where the specific energy loss measured by the ALICE Time Projection Chamber (TPC) [12] as a function of the momentum for particle (on the left) and antiparticle (right) is shown. Thus the LHC offers optimal conditions to study such nuclei. To measure the inelastic cross section of antinuclei, the different interaction of matter and antimatter with the detector is exploited. As it was shown in antideuteron studies [3], the antimatter-to-matter ratio is very sensitive to the inelastic cross sections. The ${ }^{3} \overline{\mathrm{He}} /{ }^{3} \mathrm{He}$ can be expressed as:

$$
\frac{{ }^{3} \overline{\mathrm{He}}}{{ }^{3} \mathrm{He}}=\left(\frac{{ }^{3} \overline{\mathrm{He}}}{{ }^{3} \mathrm{He}}\right)_{\text {prim }} \cdot \exp \left(-\alpha\left(\sigma_{\text {inel }}^{3 \overline{\mathrm{He}}}-\sigma_{\text {inel }}^{3}\right) \Delta x\right) .
$$

Here, $\frac{3 \overline{\mathrm{He}}}{{ }^{3} \mathrm{He}}$ is the measured ratio of the number of antihelium and helium while $\left(\frac{3 \overline{\mathrm{He}}}{{ }^{3} \mathrm{He}}\right)_{\text {prim }}$ is the primordial ratio. The traversed path length is indicated with $\Delta x$ and $\alpha=\rho N_{A} / M$, where $N_{A}$ is the Avogadro's number, $\rho$ and $M$ are the density and the molar mass of the target, respectively. The inelastic cross sections of helium and antihelium with matter are denoted as $\sigma_{\text {inel }}^{3} \overline{\mathrm{He}}$ and $\sigma_{\text {inel }}^{3} \mathrm{He}$, respectively. As the inelastic cross section of ${ }^{3} \mathrm{He}$ is rather well known, the ratio ${ }^{3} \mathrm{He} /{ }^{3} \mathrm{He}$ can be used to obtain the inelastic cross section of ${ }^{3} \overline{\mathrm{He}}$. 


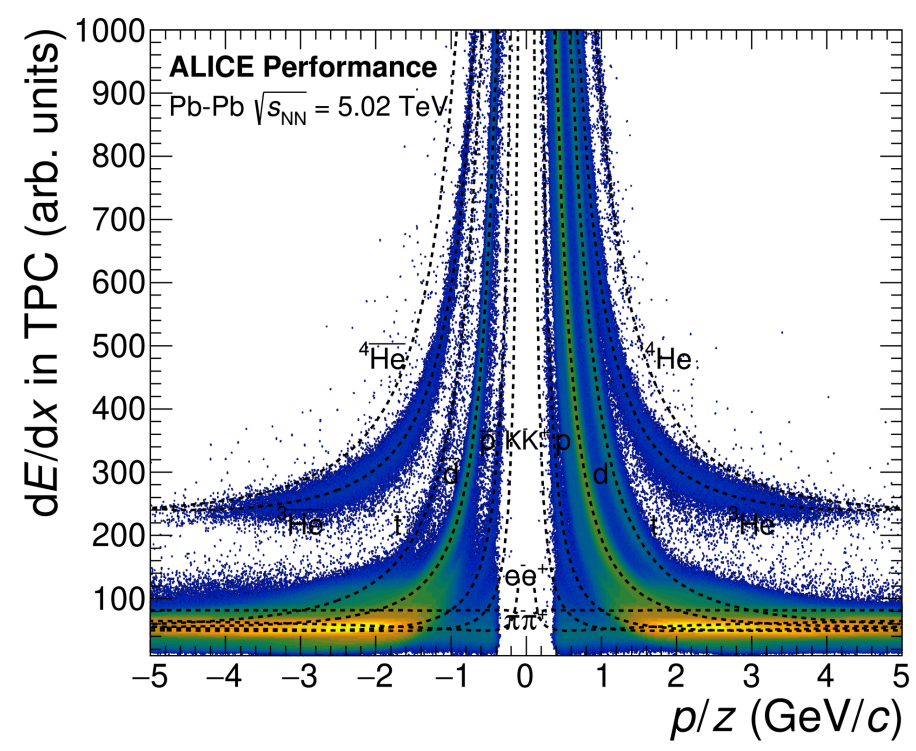

ALI-PERF-341664

Figure 1: Specific energy loss in the ALICE TPC detector as a function of rigidity.

The ${ }^{3} \overline{\mathrm{He}}$ inelastic cross section was measured using pp collisions at $\sqrt{s}=13 \mathrm{TeV}$ and $\mathrm{Pb}-\mathrm{Pb}$ collisions at $\sqrt{s_{\mathrm{NN}}}=5.02 \mathrm{TeV}$ recorded by ALICE. Here, only pp results are presented in detail. The ALICE detector was used as a target material. Full description of the experimental setup of the ALICE detector can be found in [4, 5]. The charged (anti)particles were identified by using the specific energy loss measured by the TPC. Because of the double charge of helium and antihelium, they are very well separated from the rest of the particles in such a measurement as it can be seen in Fig. 1. To select higher quality track samples, an additional hit in the Inner Tracking System (ITS) was required. The absorption probability of ${ }^{3} \overline{\mathrm{He}}$ in the detector increases with the amount of traversed material. Thus for particles with momenta $p>1 \mathrm{GeV} / c$, an additional hit in the Time-of-Flight (TOF) detector was required. The Transition Radiation Detector (TRD)[11] is in between the TPC and the TOF detectors and provides additional material budget. The measured ${ }^{3} \mathrm{He}$ spectra was corrected to account for secondary particles from spallation processes in the detector material. This correction is not needed for ${ }^{3} \overline{\mathrm{He}}$ as the probability of producing secondary ${ }^{3} \overline{\mathrm{He}}$ is extremely low. The resulting ${ }^{3} \overline{\mathrm{He}} \beta^{3} \mathrm{He}$ ratio as a function of rigidity $\left(p_{\text {prim }} / Z\right.$, where $p_{\text {prim }}$ is the momentum at primary vertex and $Z$ is the charge of the particle) is shown in Fig. 2. The red points represent Geant4 [13] simulation results while the blue points are measured ALICE results. The empty markers correspond to the ITS-TPC analysis while the full markers to the ITS-TPC-TOF analysis. The bars indicate the statistical uncertainties, while the boxes represent the systematic uncertainties. The ${ }^{3} \overline{\mathrm{He}} /{ }^{3} \mathrm{He}$ ratio in both data and $\mathrm{MC}$ simulation is smaller than unity, indicating that the inelastic cross section of the ${ }^{3} \overline{\mathrm{He}}$ is larger than that of ${ }^{3} \mathrm{He}$. The measured ${ }^{3} \overline{\mathrm{He}} /{ }^{3} \mathrm{He}$ ratio for momenta $p<1 \mathrm{GeV} / c$ is much lower than in the simulation thus a larger inelastic cross section of the ${ }^{3} \overline{\mathrm{He}}$ with respect to Geant 4 is expected.

To obtain the inelastic cross section of the ${ }^{3} \overline{\mathrm{He}}\left(\sigma_{\text {inel }}^{3 \overline{\mathrm{He}}}\right)$, the Geant 4 simulations were used. The $\sigma_{\text {inel }}^{3} \overline{\mathrm{He}}$ was varied in Geant4 bin-by-bin in momentum to reproduce the ${ }^{3} \overline{\mathrm{He}} /{ }^{3} \mathrm{He}$ ratio measured by ALICE and its corresponding $1 \sigma$ uncertainties. The resulting values represents the $\sigma_{\text {inel }}^{3 \overline{\mathrm{He}}}$ measured 


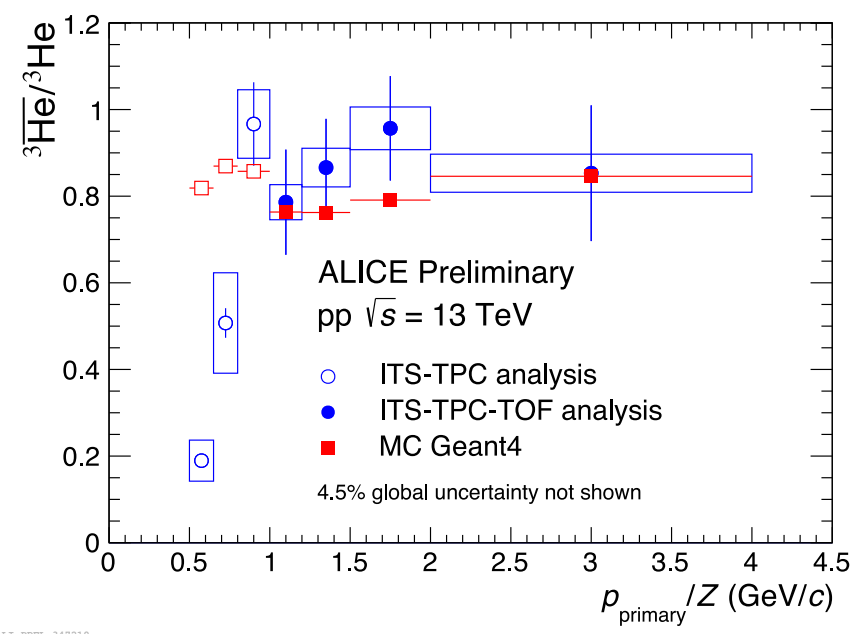

Figure 2: Raw primary ${ }^{3} \overline{\mathrm{He}} \rho^{\beta} \mathrm{He}$ ratio as a function of the momentum at the primary vertex divided by the charge.

by ALICE. The ALICE target material in the ITS-TPC analysis corresponds to a target with average charge number $Z=\langle 8.5\rangle$ and mass number $A=\langle 17.4\rangle$. In case of the ITS-TPC-TOF analysis, the corresponding values are $Z=\langle 14.8\rangle$ and $A=\langle 31.8\rangle$.

In case of the cosmic ray studies, the target nuclei in the interstellar medium are mainly protons and ${ }^{4} \mathrm{He}$. The Geant 4 simulation includes implementation of the inelastic cross section of ${ }^{3} \overline{\mathrm{He}}$ on different target nuclei [7]. We used this feature to extrapolate the measured inelastic cross section to light nuclei. A correction factor was calculated for the Geant4 inelastic cross section using the measured ${ }^{3} \overline{\mathrm{He}} \beta^{3} \mathrm{He}$ ratio. Such correction factor is momentum dependent and was assumed to be the same for all target nuclei. The ${ }^{3} \overline{\mathrm{He}}$ inelastic cross sections were estimated based on hydrogen and helium targets by applying the correction factor to the default Geant 4 implementation. A $8 \%$ uncertainty on such A scaling was assigned [7]. The resulting inelastic cross sections were implemented in GALPROP to account for the ${ }^{3} \overline{\mathrm{He}}$ annihilation in the collisions with interstellar gas in the galaxy.

\section{3. ${ }^{3} \overline{\mathrm{He}}$ Cosmic Rays}

To study how the ALICE measurement affects the cosmic ray studies, we use GALPROP to solve the transport equation:

$$
\frac{\partial \psi}{\partial t}=q(\mathbf{r}, p)+\operatorname{div}\left(D_{\mathrm{xx}} \mathbf{g r a d} \psi-\mathbf{V} \psi\right)+\frac{\partial}{\partial p} p^{2} D_{\mathrm{pp}} \frac{\partial}{\partial p} \frac{\psi}{p^{2}}-\frac{\partial}{\partial p}\left[\psi \frac{\mathrm{d} p}{\mathrm{~d} t}-\frac{p}{3}(\mathbf{d i v} \cdot \mathbf{V}) \psi\right]-\frac{\psi}{\tau} .
$$

Here, $\psi=\psi(\mathbf{r}, p, t)$ is the time dependent cosmic ray density per unit of the total particle momentum and $q(\mathbf{r}, p)$ is the ${ }^{3} \overline{\mathrm{He}}$ source function. The propagation parameters $D_{\mathrm{xx}}, \mathbf{V}$ and $D_{\mathrm{pp}}$ are the spatial diffusion coefficient, the convection velocity and the diffusive re-acceleration coefficient, respectively. The propagation parameters are expected to be the same for all particle species and were constrained using the available cosmic ray measurements. In this work, the propagation 
parameters published in [2] were used. The $-\frac{\psi}{\tau}$ term represents the particles lost via inelastic collisions with interstellar gas and is set by our ${ }^{3} \overline{\mathrm{He}}$ inelastic cross section measurement.

We implemented the ${ }^{3} \overline{\mathrm{He}}$ source functions for both dark matter annihilations and cosmic ray collisions with interstellar medium. The source function for dark matter annihilations is calculated as:

$$
q\left(\mathbf{r}, E_{\mathrm{kin}}\right)=\frac{1}{2} \frac{\rho_{\mathrm{DM}}^{2}(\mathbf{r})}{m_{\chi}^{2}}\langle\sigma v\rangle \frac{\mathrm{d} N}{\mathrm{~d} E_{\mathrm{kin}}},
$$

where $\mathbf{r}$ is the position in the Galaxy at which the source is calculated. The ${ }^{3} \overline{\mathrm{He}}$ kinetic energy and the dark matter particle mass are indicated with $E_{\text {kin }}$ and $m_{\chi}$, respectively. The velocity averaged annihilation cross section of the dark matter is denoted as $\langle\sigma v\rangle$. We use $\langle\sigma v\rangle=2.6 \cdot 10^{-26}$ $\mathrm{cm}^{3} \mathrm{~s}^{-1}[10]$. The spectrum of ${ }^{3} \overline{\mathrm{He}}$ produced in dark matter annihilation is indicated as $\mathrm{d} N / \mathrm{d} E_{\mathrm{kin}}$ and we use the spectra published in [8] for the $m_{\chi}=100 \mathrm{GeV}$ dark matter particles annihilating through $W^{+} W^{-}$and through $b \bar{b}$ channels. The Navarro-Frenk-White [6] dark matter density profile was assumed.

The ${ }^{3} \overline{\mathrm{He}}$ source function for cosmic ray collisions with the interstellar gas is expressed as:

$$
q(\mathbf{r}, p)=\sum_{\mathrm{CR}=\mathrm{p}, \mathrm{He}} \sum_{\mathrm{ISM}=\mathrm{H}, \mathrm{He}} n_{\mathrm{ISM}}(\mathbf{r}) \int d p_{\mathrm{CR}}^{\prime} \beta_{\mathrm{CR}} c \frac{d \sigma\left(p, p_{\mathrm{CR}}^{\prime}\right)}{d p} n_{\mathrm{CR}}\left(\mathbf{r}, p_{\mathrm{CR}}^{\prime}\right) .
$$

Both cosmic rays (CR) and the interstellar medium (ISM) consist mainly of protons and helium thus the pp, p-He, He-p and He-He collisions must be included. The sums over CR and ISM account for this. The density of the interstellar gas is denoted as $n_{\mathrm{ISM}}(\mathbf{r})$, while $p_{\mathrm{CR}}^{\prime}, \beta_{\mathrm{CR}}$ and $n\left(\mathbf{r}, p_{\mathrm{CR}}^{\prime}\right)$ are the momentum, the velocity and the density of the cosmic rays. $p$ is the momentum of produced ${ }^{3} \overline{\mathrm{He}} \cdot d \sigma\left(p, p^{\prime}\right) / d p$ is the ${ }^{3} \overline{\mathrm{He}}$ differential production cross section which was taken from [9].

As existing and planned cosmic ray detectors will operate inside the Solar System, one must account for the solar modulation. In this work, it was done by using the Force-Field approximation with a Fisk potential value of $0.4 \mathrm{GeV}$.

The ${ }^{3} \overline{\mathrm{He}}$ cosmic ray flux was first estimated by setting the ${ }^{3} \overline{\mathrm{He}}$ inelastic cross section to zero, thus no inelastic interactions of ${ }^{3} \overline{\mathrm{He}}$ nuclei with interstellar medium happen on their way to the Solar System. Then the inelastic cross section is set to values estimated using ALICE data and the ${ }^{3} \overline{\mathrm{He}}$ flux is reevaluated. The ratio of these fluxes defines the survival probability of ${ }^{3} \overline{\mathrm{He}}$ in the galaxy, or the transparency of our galaxy to the ${ }^{3} \overline{\mathrm{He}}$ nuclei. This approach allows us to calculate the transparency of the galaxy to antinuclei.

\section{Conclusions}

We measured the inelastic cross section of ${ }^{3} \overline{\mathrm{He}}$ using the ALICE detector as a target material. This measurement is a necessary component for indirect dark matter searches using the antinuclei cosmic rays. The described procedure to study ${ }^{3} \overline{\mathrm{He}}$ cosmic rays using GALPROP can be used to calculate the transparency of the galaxy to antinuclei. The presented ${ }^{3} \overline{\mathrm{He}}$ inelastic cross section measurement can be used as a reference in future ${ }^{3} \overline{\mathrm{He}}$ cosmic ray studies and provide experimental uncertainties from inelastic processes of ${ }^{3} \overline{\mathrm{He}}$ in the galaxy on the fluxes of ${ }^{3} \overline{\mathrm{He}}$. 


\section{References}

[1] A. Strong and I. Moskalenko, "Propagation of cosmic-ray nucleons in the galaxy", Astrophys. J.509(1998) 212-228, arXiv:astro-ph/9807150

[2] M. Boschini et al., "Inference of the Local Interstellar Spectra of Cosmic-Ray Nuclei $\mathrm{Z} \leq 28$ with the GALPROP-HelMod Framework", Astrophys. J. Suppl. 250 no. 2, (2020) 27, arXiv:2006.01337 [astro-ph.HE]

[3] ALICE Collaboration, S. Acharya et al., "Measurement of the low-energy antideuteron inelastic cross section”, Phys. Rev. Lett. 125 no. 16, (2020) 162001, arXiv:2005.11122 [nucl-ex]

[4] ALICE Collaboration, K. Aamodt et al., "The ALICE experiment at the CERN LHC”, JINST, 3 (2008) S08002

[5] ALICE Collaboration, B. B. Abelev et al., "Performance of the ALICE Experiment at the CERN LHC", Int. J. Mod. Phys. A29(2014) 1430044, arXiv:1402.4476 [nucl-ex]

[6] J. F. Navarro, C. S. Frenk, and S. D. White, "The Structure of cold dark matter halos", Astrophys. 369 J. 462(1996) 563-575, arXiv:astro-ph/9508025

[7] V. Uzhinsky, J. Apostolakis, A. Galoyan, G. Folger, V. Grichine, et al., "Antinucleus-nucleus cross sections implemented in GEANT4", Phys. Lett. B 705(2011) 235-239

[8] E. Carlson, A. Coogan, T. Linden, S. Profumo, A. Ibarra, and S. Wild, "Antihelium from Dark367Matter,’Phys. Rev. D89no. 7, (2014) 076005, arXiv:1401.2461 [hep-ph]

[9] A. Shukla, A. Datta, P. von Doetinchem, D.-M. Gomez-Coral, and C. Kanitz, "Large-scale Simulations of Antihelium Production in Cosmic-ray Interactions", Phys. Rev. D 102 no. 6, (2020) 365063004, arXiv:2006.12707 [astro-ph.HE]

[10] M. Korsmeier, F. Donato, and N. Fornengo, "Prospects to verify a possible dark matter hint in cosmic antiprotons with antideuterons and antihelium", Phys. Rev. D 97 no. 10, (2018) 103011, arXiv:1711.08465 [astro-ph.HE]

[11] ALICE Collaboration, S. Acharya et al., "The ALICE Transition Radiation Detector: construction, operation, and performance", Nucl. Instrum. Meth. A881(2018) 88-127, arXiv:1709.02743[physics.ins-det]

[12] J. Alme et al., "The ALICE TPC, a large 3-dimensional tracking device with fast readout for ultra-high multiplicity events", Nucl. Instrum. Meth.A622(2010)

[13] S. Agostinell et al., "Geant4 — a simulation toolkit", Nucl.Instrum.Meth.A 506 (2003) 250-303 


\section{Full Authors List: ALICE Collaboration}

\section{ALICE Collaboration}

S. Acharya ${ }^{143}$, D. Adamováa ${ }^{98}$, A. Adler ${ }^{76}$, G. Aglieri Rinella ${ }^{35}$, M. Agnello $^{31}$, N. Agrawal ${ }^{55}$, Z. Ahammed ${ }^{143}$, S. Ahmad ${ }^{16}$, S.U. Ahn ${ }^{78}$, I. Ahuja ${ }^{39}$, Z. Akbar ${ }^{52}$, A. Akindinov ${ }^{95}$, M. Al-Turany ${ }^{110}$, S.N. Alam ${ }^{16,41}$, D. Aleksandrov ${ }^{91}$, B. Alessandro ${ }^{61}$, H.M. Alfanda ${ }^{7}$, R. Alfaro Molina ${ }^{73}$, B. Ali ${ }^{16}$, Y. Ali ${ }^{14}$, A. Alici ${ }^{26}$, N. Alizadehvandchali ${ }^{127}$, A. Alkin ${ }^{35}$, J. Alme ${ }^{21}$, T. Alt ${ }^{70}$, L. Altenkamper ${ }^{21}$, I. Altsybeev ${ }^{115}$, M.N. Anaam ${ }^{7}$, C. Andrei ${ }^{49}$, D. Andreou ${ }^{93}$, A. Andronic ${ }^{146}$, M. Angeletti ${ }^{35}$, V. Anguelov ${ }^{107}$, F. Antinori ${ }^{58}$, P. Antonioli ${ }^{55}$, C. Anuj ${ }^{16}$, N. Apadula ${ }^{82}$, L. Aphecetche ${ }^{117}$, H. Appelshäuser $^{70}$, S. Arcelli ${ }^{26}$, R. Arnaldi ${ }^{61}$, I.C. Arsene ${ }^{20}$, M. Arslandok ${ }^{148,107}$, A. Augustinus ${ }^{35}$, R. Averbeck ${ }^{110}$, S. Aziz ${ }^{80}$, M.D. Azmi ${ }^{16}$, A. Badalà ${ }^{57}$, Y.W. Baek ${ }^{42}$, X. Bai ${ }^{131,110}$, R. Bailhache ${ }^{70}$, Y. Bailung ${ }^{51}$, R. Bala ${ }^{104}$, A. Balbino ${ }^{31}$, A. Baldisseri ${ }^{140}$, B. Balis ${ }^{2}$, M. Ball ${ }^{44}$, D. Banerjee ${ }^{4}$, R. Barbera ${ }^{27}$, L. Barioglio ${ }^{108}$, M. Barlou ${ }^{87}$, G.G. Barnaföldi ${ }^{147}$, L.S. Barnby ${ }^{97}$, V. Barret ${ }^{137}$, C. Bartels ${ }^{130}$, K. Barth ${ }^{35}$, E. Bartsch ${ }^{70}$, F. Baruffaldi ${ }^{28}$, N. Bastid ${ }^{137}$, S. Basu ${ }^{83}$, G. Batigne ${ }^{117}$, B. Batyunya ${ }^{77}$, D. Bauri ${ }^{50}$, J.L. Bazo Alba ${ }^{114}$, I.G. Bearden ${ }^{92}$, C. Beattie ${ }^{148}$, I. Belikov ${ }^{139}$, A.D.C. Bell Hechavarria ${ }^{146}$, F. Bellini ${ }^{26}$, R. Bellwied ${ }^{127}$, S. Belokurova ${ }^{115}$, V. Belyaev ${ }^{96}$, G. Bencedi ${ }^{71}$, S. Beole $^{25}$, A. Bercuci ${ }^{49}$, Y. Berdnikov ${ }^{101}$, A. Berdnikova ${ }^{107}$, L. Bergmann ${ }^{107}$, M.G. Besoiu ${ }^{69}$, L. Betev ${ }^{35}$, P.P. Bhaduri ${ }^{143}$, A. Bhasin ${ }^{104}$, I.R. Bhat ${ }^{104}$, M.A. Bhat ${ }^{4}$, B. Bhattacharjee ${ }^{43}$, P. Bhattacharya ${ }^{23}$, L. Bianchi ${ }^{25}$, N. Bianchii ${ }^{53}$, J. Bielčík ${ }^{38}$, J. Bielčíková ${ }^{98}$, J. Biernat ${ }^{120}$, A. Bilandzic ${ }^{108}$, G. Biro ${ }^{147}$, S. Biswas ${ }^{4}$, J.T. Blair ${ }^{121}$, D. Blau ${ }^{91,84}$, M.B. Blidaru ${ }^{110}$, C. Blume ${ }^{70}$, G. Boca ${ }^{29,59}$, F. Bock ${ }^{99}$, A. Bogdanov ${ }^{96}$, S. Boi ${ }^{23}$, J. Bok ${ }^{63}$, L. Boldizsár ${ }^{147}$, A. Bolozdynya ${ }^{96}$, M. Bombara ${ }^{39}$, P.M. Bond ${ }^{35}$, G. Bonomi ${ }^{142,59}$, H. Borel ${ }^{140}$, A. Borissov ${ }^{84}$, H. Bossi ${ }^{148}$, E. Botta ${ }^{25}$, L. Bratrud ${ }^{70}$, P. Braun-Munzinger ${ }^{110}$, M. Bregant ${ }^{123}$, M. Broz ${ }^{38}$, G.E. Bruno ${ }^{109,34}$, M.D. Buckland ${ }^{130}$, D. Budnikov ${ }^{111}$, H. Buesching ${ }^{70}$, S. Bufalino ${ }^{31}$, O. Bugnon ${ }^{117}$, P. Buhler ${ }^{116}$, Z. Buthelezi ${ }^{74,134}$, J.B. Butt ${ }^{14}$, A. Bylinkin ${ }^{129}$, S.A. Bysiak ${ }^{120}$, M. Cai ${ }^{28,7}$, H. Caines ${ }^{148}$, A. Caliva ${ }^{110}$, E. Calvo Villar ${ }^{114}$, J.M.M. Camacho ${ }^{122}$, R.S. Camacho ${ }^{46}$, P. Camerini ${ }^{24}$, F.D.M. Canedo ${ }^{123}$, F. Carnesecchi ${ }^{35,26}$, R. Caron ${ }^{140}$, J. Castillo Castellanos ${ }^{140}$, E.A.R. Casula ${ }^{23}$, F. Catalano ${ }^{31}$, C. Ceballos Sanchez ${ }^{77}$, P. Chakraborty ${ }^{50}$, S. Chandra ${ }^{143}$, S. Chapeland ${ }^{35}$, M. Chartier ${ }^{130}$, S. Chattopadhyay ${ }^{143}$, S. Chattopadhyay ${ }^{112}$, A. Chauvin ${ }^{23}$, T.G. Chavez ${ }^{46}$, T. Cheng ${ }^{7}$, C. Cheshkov ${ }^{138}$, B. Cheynis ${ }^{138}$, V. Chibante Barroso ${ }^{35}$, D.D. Chinellato ${ }^{124}$, S. Cho $^{63}$, P. Chochula ${ }^{35}$, P. Christakoglou ${ }^{93}$, C.H. Christensen ${ }^{92}$, P. Christiansen ${ }^{83}$, T. Chujo ${ }^{136}$, C. Cicalo ${ }^{56}$, L. Cifarellii ${ }^{26}$, F. Cindolo ${ }^{55}$, M.R. Ciupek ${ }^{110}$, G. Clai ${ }^{\mathrm{II}, 55}$, J. Cleymans ${ }^{\mathrm{I}}{ }^{126}$, F. Colamaria ${ }^{54}$, J.S. Colburn ${ }^{113}$, D. Colella ${ }^{109,54,34,147}$, A. Collu ${ }^{82}$, M. Colocci ${ }^{35}$, M. Concas ${ }^{I I I, 61}$, G. Conesa Balbastre ${ }^{81}$, Z. Conesa del Valle ${ }^{80}$, G. Contin ${ }^{24}$, J.G. Contreras ${ }^{38}$, M.L. Coquet ${ }^{140}$, T.M.Cormier ${ }^{99}$, P. Cortese ${ }^{32}$, M.R. Cosentino ${ }^{125}$, F. Costa $^{35}$, S. Costanza ${ }^{29,59}$, P. Crochet ${ }^{137}$, R. Cruz-Torres ${ }^{82}$, E. Cuautle ${ }^{71}$, P. Cui $^{7}$, L. Cunqueiro ${ }^{99}$, A. Dainese ${ }^{58}$, M.C. Danisch ${ }^{107}$, A. Danu ${ }^{69}$, I. Das ${ }^{112}$, P. Das ${ }^{89}$, P. Das ${ }^{4}$, S. Das ${ }^{4}$, S. Dash ${ }^{50}$, S. De $^{89}$, A. De Caro ${ }^{30}$, G. de Cataldo ${ }^{54}$, L. De Cilladi ${ }^{25}$, J. de Cuveland ${ }^{40}$, A. De Falco ${ }^{23}$, D. De Gruttola ${ }^{30}$, N. De $\mathrm{Marco}^{61}$, C. De Martinn ${ }^{24}$, S. De Pasquale ${ }^{30}$, S. Deb ${ }^{51}$, H.F. Degenhardt ${ }^{123}$, K.R. Deja ${ }^{144}$, L. Dello Stritto ${ }^{30}$, S. Delsanto ${ }^{25}$, W. Deng ${ }^{7}$, P. Dhankher ${ }^{19}$, D. Di Bari ${ }^{34}$, A. Di Mauro ${ }^{35}$, R.A. Diaz ${ }^{8}$, T. Dietel ${ }^{126}$, Y. Ding ${ }^{138,7}$, R. Divià ${ }^{35}$, D.U. Dixit ${ }^{19}, \varnothing$. Djuvsland $^{21}$, U. Dmitrieva ${ }^{65}$, J. Do ${ }^{63}$, A. Dobrin ${ }^{69}$, B. Dönigus ${ }^{70}$, O. Dordic ${ }^{20}$, A.K. Dubey ${ }^{143}$, A. Dubla ${ }^{110,93}$, S. Dudi ${ }^{103}$, M. Dukhishyam ${ }^{89}$, P. Dupieux ${ }^{137}$, N. Dzalaiova ${ }^{13}$, T.M. Eder ${ }^{146}$, R.J. Ehlers ${ }^{99}$, V.N. Eikeland ${ }^{21}$, F. Eisenhut ${ }^{70}$, D. Elia $^{54}$, B. Erazmus ${ }^{117}$, F. Ercolessi ${ }^{26}$, F. Erhardt ${ }^{102}$, A. Erokhin ${ }^{115}$, M.R. Ersdal ${ }^{21}$, 
B. Espagnon ${ }^{80}$, G. Eulisse ${ }^{35}$, D. Evans ${ }^{113}$, S. Evdokimov ${ }^{94}$, L. Fabbietti ${ }^{108}$, M. Faggin ${ }^{28}$, J. Faivre ${ }^{81}$, F. Fan ${ }^{7}$, A. Fantoni ${ }^{53}$, M. Fasel ${ }^{99}$, P. Fecchio ${ }^{31}$, A. Feliciello ${ }^{61}$, G. Feofilov ${ }^{115}$, A. Fernández Téllez $^{46}$, A. Ferrero ${ }^{140}$, A. Ferretti ${ }^{25}$, V.J.G. Feuillard ${ }^{107}$, J. Figiel ${ }^{120}$, S. Filchagin ${ }^{111}$, D. Finogeev ${ }^{65}$, F.M. Fionda ${ }^{56,21}$, G. Fiorenza ${ }^{35,109}$, F. Flor ${ }^{127}$, A.N. Flores ${ }^{121}$, S. Foertsch ${ }^{74}$, P. Foka ${ }^{110}$, S. Fokin ${ }^{91}$, E. Fragiacomo ${ }^{62}$, E. Frajna ${ }^{147}$, U. Fuchs ${ }^{35}$, N. Funicello ${ }^{30}$, C. Furget ${ }^{81}$, A. Furs ${ }^{65}$, J.J. Gaardhøje ${ }^{92}$, M. Gagliardi ${ }^{25}$, A.M. Gago ${ }^{114}$, A. Gal ${ }^{139}$, C.D. Galvan ${ }^{122}$, P. Ganoti ${ }^{87}$, C. Garabatos ${ }^{110}$, J.R.A. Garcia ${ }^{46}$, E. Garcia-Solis ${ }^{10}$, K. Garg ${ }^{117}$, C. Gargiulo ${ }^{35}$, A. Garibli ${ }^{90}$, K. Garner ${ }^{146}$, P. Gasik ${ }^{110}$, E.F. Gauger ${ }^{121}$, A. Gautam ${ }^{129}$, M.B. Gay Ducati ${ }^{72}$, M. Germain ${ }^{117}$, P. Ghosh ${ }^{143}$, S.K. Ghosh ${ }^{4}$, M. Giacalone ${ }^{26}$, P. Gianotti ${ }^{53}$, P. Giubellino ${ }^{110,61}$, P. Giubilato ${ }^{28}$, A.M.C. Glaenzer ${ }^{140}$, P. Glässel ${ }^{107}$, D.J.Q. Goh ${ }^{85}$, V. Gonzalez ${ }^{145}$, L.H. González-Trueba ${ }^{73}$, S. Gorbunov ${ }^{40}$, M. Gorgon ${ }^{2}$, L. Görlich ${ }^{120}$, S. Gotovac $^{36}$, V. Grabski ${ }^{73}$, L.K. Graczykowski ${ }^{144}$, L. Greiner ${ }^{82}$, A. Grelli ${ }^{64}$, C. Grigoras ${ }^{35}$, V. Grigoriev ${ }^{96}$, S. Grigoryan ${ }^{77,1}$, O.S. Groettvik ${ }^{21}$, F. Grosa ${ }^{35,61}$, J.F. Grosse-Oetringhaus ${ }^{35}$, R. Grosso ${ }^{110}$, G.G. Guardiano ${ }^{124}$, R. Guernane ${ }^{81}$, M. Guilbaud ${ }^{117}$, K. Gulbrandsen ${ }^{92}$, T. Gunji ${ }^{135}$, W. Guo ${ }^{7}$, A. Gupta ${ }^{104}$, R. Gupta ${ }^{104}$, S.P. Guzman ${ }^{46}$, L. Gyulai ${ }^{147}$, M.K. Habib ${ }^{110}$, C. Hadjidakis ${ }^{80}$, G. Halimoglu ${ }^{70}$, H. Hamagaki ${ }^{85}$, G. Hamar ${ }^{147}$, M. Hamid ${ }^{7}$, R. Hannigan ${ }^{121}$, M.R. Haque ${ }^{144,89}$, A. Harlenderova ${ }^{110}$, J.W. Harris ${ }^{148}$, A. Harton ${ }^{10}$, J.A. Hasenbichler ${ }^{35}$, H. Hassan ${ }^{99}$, D. Hatzifotiadou ${ }^{55}$, P. Hauer ${ }^{44}$, L.B. Havener ${ }^{148}$, S. Hayashi ${ }^{135}$, S.T. Heckel ${ }^{108}$, E. Hellbär ${ }^{110}$, H. Helstrup ${ }^{37}$, T. Herman ${ }^{38}$, E.G. Hernandez ${ }^{46}$, G. Herrera Corral ${ }^{9}$, F. Herrmann ${ }^{146}$, K.F. Hetland ${ }^{37}$, H. Hillemanns ${ }^{35}$, C. Hills ${ }^{130}$, B. Hippolyte ${ }^{139}$, B. Hofman ${ }^{64}$, B. Hohlweger ${ }^{93}$, J. Honermann ${ }^{146}$, G.H. Hong ${ }^{149}$, D. Horak ${ }^{38}$, A. Horzyk ${ }^{2}$, R. Hosokawa ${ }^{15}$,

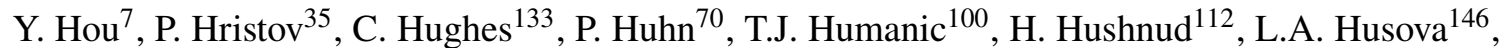
A. Hutson ${ }^{127}$, D. Hutter ${ }^{40}$, J.P. Iddon ${ }^{35,130}$, R. Ilkaev ${ }^{111}$, H. Ilyas ${ }^{14}$, M. Inaba ${ }^{136}$, G.M. Innocenti ${ }^{35}$, M. Ippolitov ${ }^{91}$, A. Isakov ${ }^{38,98}$, M.S. Islam ${ }^{112}$, M. Ivanov ${ }^{110}$, V. Ivanov ${ }^{101}$, V. Izucheev ${ }^{94}$, M. Jablonski ${ }^{2}$, B. Jacak ${ }^{82}$, N. Jacazio ${ }^{35}$, P.M. Jacobs ${ }^{82}$, S. Jadlovska ${ }^{119}$, J. Jadlovsky ${ }^{119}$, S. Jaelani ${ }^{64}$, C. Jahnke ${ }^{124,123}$, M.J. Jakubowska ${ }^{144}$, A. Jalotra ${ }^{104}$, M.A. Janik ${ }^{144}$, T. Janson ${ }^{76}$, M. Jercic ${ }^{102}$, O. Jevons ${ }^{113}$, A.A.P. Jimenez ${ }^{71}$, F. Jonas ${ }^{99,146}$, P.G. Jones ${ }^{113}$, J.M. Jowett ${ }^{35,110}$, J. Jung ${ }^{70}$, M. Jung ${ }^{70}$, A. Junique ${ }^{35}$, A. Jusko ${ }^{113}$, J. Kaewjai ${ }^{118}$, P. Kalinak ${ }^{66}$, A.S. Kalteyer ${ }^{110}$, A. Kalweit ${ }^{35}$, V. Kaplin ${ }^{96}$, S. Kar ${ }^{7}$, A. Karasu Uysal $^{79}$, D. Karatovic ${ }^{102}$, O. Karavichev ${ }^{65}$, T. Karavicheva ${ }^{65}$, P. Karczmarczyk ${ }^{144}$, E. Karpechev ${ }^{65}$, A. Kazantsev ${ }^{91}$, U. Kebschull ${ }^{76}$, R. Keidel ${ }^{48}$, D.L.D. Keijdener ${ }^{64}$, M. Keil ${ }^{35}$, B. Ketzer ${ }^{44}$,Z. Khabanova ${ }^{93}$, A.M. Khan ${ }^{7}$, S. Khan ${ }^{16}$, A. Khanzadeev ${ }^{101}$, Y. Kharlov ${ }^{94,84}$, A. Khatun ${ }^{16}$, A. Khuntia ${ }^{120}$, B. Kileng ${ }^{37}$, B. $\operatorname{Kim}^{17,63}$, C. Kim ${ }^{17}$, D.J. Kim ${ }^{128}$, E.J. Kim ${ }^{75}$, J. Kim ${ }^{149}$, J.S. Kim ${ }^{42}$, J. Kim ${ }^{107}$, J. Kim ${ }^{149}$, J. Kim ${ }^{75}$, M. Kim ${ }^{107}$, S. Kim ${ }^{18}$, T. Kim ${ }^{149}$, S. Kirsch ${ }^{70}$, I. Kisel ${ }^{40}$, S. Kiselev ${ }^{95}$, A. Kisiel ${ }^{144}$, J.P. Kitowski ${ }^{2}$, J.L. Klay ${ }^{6}$, J. Klein ${ }^{35}$, S. Klein ${ }^{82}$, C. Klein-Bösing ${ }^{146}$, M. Kleiner ${ }^{70}$, T. Klemenz ${ }^{108}$, A. Kluge ${ }^{35}$, A.G. Knospe ${ }^{127}$, C. Kobdaj ${ }^{18}$, M.K. Köhler ${ }^{107}$, T. Kollegger ${ }^{110}$, A. Kondratyev ${ }^{77}$, N. Kondratyeva ${ }^{96}$, E. Kondratyuk ${ }^{94}$, J. Konig ${ }^{70}$, S.A. Konigstorfer ${ }^{108}$, P.J. Konopka ${ }^{35,2}$, G. Kornakov ${ }^{144}$, S.D. Koryciak ${ }^{2}$, L. Koska ${ }^{119}$, A. Kotliarov ${ }^{98}$, O. Kovalenko ${ }^{88}$, V. Kovalenko ${ }^{115}$, M. Kowalski ${ }^{120}$, I. Králik ${ }^{66}$, A. Kravčákováa ${ }^{39}$, L. Kreis ${ }^{110}$, M. Krivda ${ }^{113,66}$, F. Krizek ${ }^{98}$, K. Krizkova Gajdosova ${ }^{38}$, M. Kroesen ${ }^{107}$, M. Krüger ${ }^{70}$, E. Kryshen ${ }^{101}$, M. Krzewicki ${ }^{40}$, V. Kučera ${ }^{35}$, C. Kuhn ${ }^{139}$, P.G. Kuijer ${ }^{93}$, T. Kumaoka ${ }^{136}$, D. Kumarr ${ }^{143}$, L. Kumar ${ }^{103}$, N. Kumar ${ }^{103}$, S. Kundu ${ }^{35}$, P. Kurashvili ${ }^{88}$, A. Kurepin ${ }^{65}$, A.B. Kurepin ${ }^{65}$, A. Kuryakin ${ }^{111}$, S. Kushpil ${ }^{98}$, J. Kvapil ${ }^{113}$, M.J. Kweon ${ }^{63}$, J.Y. Kwon ${ }^{63}$, Y. Kwon ${ }^{149}$, S.L. La Pointe ${ }^{40}$, P. La Rocca ${ }^{27}$, Y.S. Lai ${ }^{82}$, A. Lakrathok ${ }^{118}$, M. Lamanna ${ }^{35}$, R. Langoy ${ }^{132}$, K. Lapidus ${ }^{35}$, P. Larionov ${ }^{35,53}$, E. Laudi ${ }^{35}$, L. Lautner ${ }^{35,108}$, R. Lavicka ${ }^{38}$, T. Lazareva ${ }^{115}$, R. Lea ${ }^{142,24,59}$, J. Lehrbach ${ }^{40}$, R.C. Lemmon ${ }^{97}$, I. León Monzón ${ }^{122}$, E.D. Lesser ${ }^{19}$, M. Lettrich ${ }^{35,108}$, P. Lévai ${ }^{147}$,

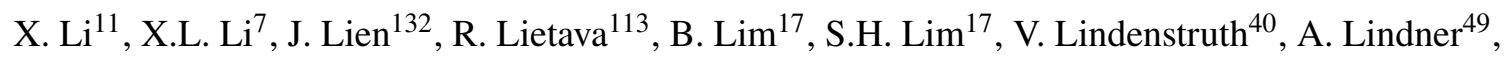


C. Lippmann ${ }^{110}$, A. Liu ${ }^{19}$, D.H. Liu ${ }^{7}$, J. Liu ${ }^{130}$, I.M. $\operatorname{Lofnes}^{21}$, V. Loginov ${ }^{96}$, C. Loizides ${ }^{99}$, P. Loncar ${ }^{36}$, J.A. Lopez ${ }^{107}$, X. Lopez ${ }^{137}$, E. López Torres ${ }^{8}$, J.R. Luhder ${ }^{146}$, M. Lunardon ${ }^{28}$, G. Luparello ${ }^{62}$, Y.G. Ma ${ }^{41}$, A. Maevskaya ${ }^{65}$, M. Mager ${ }^{35}$, T. Mahmoud ${ }^{44}$, A. Maire ${ }^{139}$, M. Malaev ${ }^{101}$, N.M. Malik ${ }^{104}$, Q.W. Malik ${ }^{20}$, L. Malinina ${ }^{\mathrm{IV}, 77}$, D. Mal'Kevich ${ }^{95}$, N. Mallick ${ }^{51}$, P. Malzacher ${ }^{110}$, G. Mandaglio $^{33,57}$, V. Manko ${ }^{91}$, F. Manso ${ }^{137}$, V. Manzari ${ }^{54}$, Y. Mao ${ }^{7}$, J. Mareš ${ }^{68}$, G.V. Margagliotti ${ }^{24}$, A. Margotti ${ }^{55}$, A. Marín ${ }^{110}$, C. Markert ${ }^{121}$, M. Marquard ${ }^{70}$, N.A. Martin ${ }^{107}$, P. Martinengo ${ }^{35}$, J.L. Martinez ${ }^{127}$, M.I. Martínez ${ }^{46}$, G. Martínez García ${ }^{117}$, S. Masciocchi ${ }^{110}$, M. Masera ${ }^{25}$, A. Masoni ${ }^{56}$, L. Massacrier ${ }^{80}$, A. Mastroserio ${ }^{141,54}$, A.M. Mathis ${ }^{108}$, O. Matonoha ${ }^{83}$, P.F.T. Matuoka ${ }^{123}$, A. Matyja ${ }^{120}$, C. Mayer ${ }^{120}$, A.L. Mazuecos ${ }^{35}$, F. Mazzaschi ${ }^{25}$, M. Mazzilli ${ }^{35}$, M.A. Mazzoni ${ }^{\mathrm{I}, 60}$, J.E. Mdhluli ${ }^{134}$, A.F. Mechler $^{70}$, F. Meddi ${ }^{22}$, Y. Melikyan ${ }^{65}$, A. Menchaca-Rocha ${ }^{73}$, E. Meninno ${ }^{116,30}$, A.S. Menon ${ }^{127}$, M. Meres ${ }^{13}$, S. Mhlanga ${ }^{126,74}$, Y. Miake ${ }^{136}$, L. Micheletti ${ }^{61,25}$, L.C. Migliorin ${ }^{138}$, D.L. Mihaylov ${ }^{108}$, K. Mikhaylov ${ }^{77,95}$, A.N. Mishra ${ }^{147}$, D. Miśkowiec ${ }^{110}$, A. Modak $^{4}$, A.P. Mohanty ${ }^{64}$, B. Mohanty ${ }^{89}$, M. Mohisin Khan ${ }^{\text {V,16, M.A. Molander }}{ }^{45}$, Z. Moravcova ${ }^{92}$, C. Mordasini ${ }^{108}$, D.A. Moreira De Godoy $^{146}$, L.A.P. Moreno ${ }^{46}$, I. Morozov ${ }^{65}$, A. Morsch ${ }^{35}$, T. Mrnjavac ${ }^{35}$, V. Muccifora ${ }^{53}$, E. Mudnic ${ }^{36}$, D. Mühlheim ${ }^{146}$, S. Muhuri ${ }^{143}$, J.D. Mulligan ${ }^{82}$, A. Mulliri' ${ }^{23}$, M.G. Munhoz ${ }^{123}$, R.H. Munzer ${ }^{70}$, H. Murakami ${ }^{135}$, S. Murray ${ }^{126}$, L. Musa ${ }^{35}$, J. Musinsky ${ }^{66}$, J.W. Myrcha ${ }^{144}$, B. Naik ${ }^{134,50}$, R. Nair ${ }^{88}$, B.K. Nandi ${ }^{50}$, R. Nania ${ }^{55}$, E. Nappi ${ }^{54}$, A.F. Nassirpour ${ }^{83}$, A. Nath ${ }^{107}$, C. Nattrass ${ }^{133}$, A. $\mathrm{Neagu}^{20}$, L. Nellen $^{71}$, S.V. Nesbo ${ }^{37}$, G. Neskovic ${ }^{40}$, D. Nesterov ${ }^{115}$, B.S. Nielsen ${ }^{92}$, S. Nikolaev ${ }^{91}$, S. Nikulin ${ }^{91}$, V. Nikulin ${ }^{101}$, F. Noferini ${ }^{55}$, S. Noh ${ }^{12}$, P. Nomokonov ${ }^{77}$, J. Norman ${ }^{130}$, N. Novitzky ${ }^{136}$, P. Nowakowski ${ }^{144}$, A. Nyanin ${ }^{91}$, J. Nystrand ${ }^{21}$, M. Ogino ${ }^{85}$, A. Ohlson ${ }^{83}$, V.A. Okorokov ${ }^{96}$, J. Oleniacz ${ }^{144}$, A.C. Oliveira Da Silva $^{133}$, M.H. Oliver ${ }^{148}$, A. Onnerstad ${ }^{128}$, C. Oppedisano ${ }^{61}$, A. Ortiz Velasquez ${ }^{71}$, T. Osako ${ }^{47}$, A. Oskarsson ${ }^{83}$, J. Otwinowski ${ }^{120}$, M. Oya ${ }^{47}$, K. Oyama $^{85}$, Y. Pachmayer ${ }^{107}$, S. Padhan $^{50}$, D. Pagano ${ }^{142,59}$, G. Paić ${ }^{71}$, A. Palasciano ${ }^{54}$, J. Pan ${ }^{145}$, S. Panebianco ${ }^{140}$, P. Pareek ${ }^{143}$, J. Park ${ }^{63}$, J.E. Parkkila ${ }^{128}$, S.P. Pathak ${ }^{127}$, R.N. Patra ${ }^{104,35}$, B. Paul ${ }^{23}$, H. Pei ${ }^{7}$, T. Peitzmann ${ }^{64}$, X. Peng ${ }^{7}$, L.G. Pereira ${ }^{72}$, H. Pereira Da Costa ${ }^{140}$, D. Peresunko ${ }^{91,84}$, G.M. Perez ${ }^{8}$, S. Perrin ${ }^{140}$, Y. Pestov ${ }^{5}$, V. Petráček ${ }^{38}$, M. Petrovici ${ }^{49}$, R.P. Pezzi ${ }^{117,72}$, S. Piano ${ }^{62}$, M. Pikna ${ }^{13}$, P. Pillot ${ }^{117}$, O. Pinazza ${ }^{55,35}$, L. Pinsky ${ }^{127}$, C. Pinto ${ }^{27}$, S. Pisano ${ }^{53}$, M. Płoskoń ${ }^{82}$, M. Planinic ${ }^{102}$, F. Pliquett ${ }^{70}$, M.G. Poghosyan ${ }^{99}$, B. Polichtchouk ${ }^{94}$, S. Politano ${ }^{31}$, N. Poljak ${ }^{102}$, A. Pop ${ }^{49}$, S. Porteboeuf-Houssais ${ }^{137}$, J. Porter ${ }^{82}$, V. Pozdniakov ${ }^{77}$, S.K. Prasad ${ }^{4}$, R. Preghenella ${ }^{55}$, F. Prino ${ }^{61}$, C.A. Pruneau ${ }^{145}$, I. Pshenichnov ${ }^{65}$, M. Puccio ${ }^{35}$, S. Qiu ${ }^{93}$, L. Quaglia ${ }^{25}$, R.E. Quishpe ${ }^{127}$, S. Ragoni ${ }^{113}$, A. Rakotozafindrabe ${ }^{140}$, L. Ramello ${ }^{32}$, F. Rami ${ }^{139}$, S.A.R. Ramirez ${ }^{46}$, A.G.T. Ramos ${ }^{34}$, T.A. Rancien ${ }^{81}$, R. Raniwala ${ }^{105}$, S. Raniwala ${ }^{105}$,

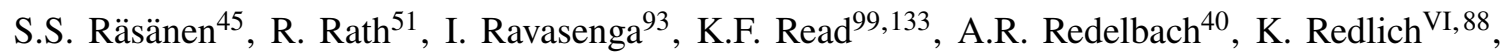
A. Rehman ${ }^{21}$, P. Reichelt ${ }^{70}$, F. Reidt ${ }^{35}$, H.A. Reme-ness ${ }^{37}$, R. Renfordt $^{70}$, Z. Rescakova $^{39}$, K. Reygers ${ }^{107}$, A. Riabov ${ }^{101}$, V. Riabov ${ }^{101}$, T. Richert ${ }^{83}$, M. Richter ${ }^{20}$, W. Riegler ${ }^{35}$, F. Riggi ${ }^{27}$, C. Ristea ${ }^{69}$, M. Rodríguez Cahuantzi $^{46}$, K. Røed ${ }^{20}$, R. Rogalev ${ }^{94}$, E. Rogochaya ${ }^{77}$, T.S. Rogoschinski ${ }^{70}$, D. Rohr ${ }^{35}$, D. Röhrich ${ }^{21}$, P.F. Rojas ${ }^{46}$, P.S. Rokita ${ }^{144}$, F. Ronchetti ${ }^{53}$, A. Rosano ${ }^{33,57}$, E.D. Rosas ${ }^{71}$, A. Rossi ${ }^{58}$, A. Rotondi ${ }^{29,59}$, A. Roy ${ }^{51}$, P. Roy ${ }^{112}$, S. Roy ${ }^{50}$, N. Rubini ${ }^{26}$, O.V. Rueda ${ }^{83}$, R. Rui $^{24}$, B. Rumyantsev ${ }^{77}$, P.G. Russek ${ }^{2}$, A. Rustamov ${ }^{90}$, E. Ryabinkin ${ }^{91}$, Y. Ryabov ${ }^{101}$, A. Rybicki120, H. Rytkonen ${ }^{128}$, W. Rzesa ${ }^{144}$, O.A.M. Saarimaki ${ }^{45}$, R. Sadek ${ }^{117}$, S. Sadovsky ${ }^{94}$, J. Saetre ${ }^{21}$, K. Šafař́k ${ }^{38}$, S.K. Saha ${ }^{143}$, S. Saha ${ }^{89}$, B. Sahoo ${ }^{50}$, P. Sahoo ${ }^{50}$, R. Sahoo ${ }^{51}$, S. Sahoo ${ }^{67}$, D. Sahu ${ }^{51}$, P.K. Sahu ${ }^{67}$, J. Saini ${ }^{143}$, S. Sakai ${ }^{136}$, M.P. Salvan ${ }^{110}$, S. Sambyal ${ }^{104}$, V. Samsonov ${ }^{\text {I,101,96, D. Sarkar }}{ }^{145}$, N. Sarkar ${ }^{143}$, P. Sarma ${ }^{43}$, V.M. Sarti ${ }^{108}$, M.H.P. Sas ${ }^{148}$, J. Schambach ${ }^{99,121}$, H.S. Scheid ${ }^{70}$, C. Schiaua ${ }^{49}$, R. Schicker ${ }^{107}$, A. Schmah ${ }^{107}$, C. Schmidt ${ }^{110}$, H.R. Schmidt ${ }^{106}$, M.O. Schmidt ${ }^{35}$, M. Schmidt ${ }^{106}$, N.V. Schmidt ${ }^{99,70,}$ 
A.R. Schmier ${ }^{133}$, R. Schotter ${ }^{139}$, J. Schukraft ${ }^{35}$, Y. Schutz ${ }^{139}$, K. Schwarz ${ }^{110}$, K. Schweda ${ }^{110}$, G. Scioli ${ }^{26}$, E. Scomparin ${ }^{61}$, J.E. Seger ${ }^{15}$, Y. Sekiguchi ${ }^{135}$, D. Sekihata ${ }^{135}$, I. Selyuzhenkov ${ }^{110,96}$, S. Senyukov ${ }^{139}$, J.J. Seo ${ }^{63}$, D. Serebryakov ${ }^{65}$, L. Šerkšnytėe8, A. Sevcenco ${ }^{69}$, T.J. Shaba ${ }^{74}$, A. Shabanov ${ }^{65}$, A. Shabetai ${ }^{117}$, R. Shahoyan ${ }^{35}$, W. Shaikh ${ }^{112}$, A. Shangaraev ${ }^{94}$, A. Sharma ${ }^{103}$, H. Sharma ${ }^{120}$, M. Sharma ${ }^{104}$, N. Sharma ${ }^{103}$, S. Sharma ${ }^{104}$, U. Sharma ${ }^{104}$, O. Sheibani ${ }^{127}$, K. Shigaki ${ }^{47}$, M. Shimomura ${ }^{86}$, S. Shirinkin ${ }^{95}$, Q. Shou ${ }^{41}$, Y. Sibiriak ${ }^{91}$, S. Siddhanta ${ }^{56}$, T. Siemiarczuk ${ }^{88}$, T.F. Silva ${ }^{123}$, D. Silvermyr ${ }^{83}$, T. Simantathammakul ${ }^{118}$, G. Simonetti ${ }^{35}$, B. Singh ${ }^{108}$, R. Singh ${ }^{89}$, R. Singh ${ }^{104}$, R. Singh ${ }^{51}$, V.K. Singh ${ }^{143}$, V. Singhal ${ }^{143}$, T. Sinha ${ }^{112}$, B. Sitar ${ }^{13}$, M. Sitta ${ }^{32}$, T.B. Skaali ${ }^{20}$, G. Skorodumovs ${ }^{107}$, M. Slupecki ${ }^{45}$, N. Smirnov ${ }^{148}$, R.J.M. Snellings ${ }^{64}$, C. Soncco ${ }^{114}$, J. Song ${ }^{127}$, A. Songmoolnak ${ }^{118}$, F. Soramel ${ }^{28}$, S. Sorensen ${ }^{133}$, I. Sputowska ${ }^{120}$, J. Stachel ${ }^{107}$, I. Stan ${ }^{69}$, P.J. Steffanic ${ }^{133}$, S.F. Stiefelmaier ${ }^{107}$, D. Stocco ${ }^{117}$, I. Storehaug ${ }^{20}$, M.M. Storetvedt ${ }^{37}$, C.P. Stylianidis ${ }^{93}$, A.A.P. Suaide ${ }^{123}$, T. Sugitate ${ }^{47}$, C. Suire ${ }^{80}$, M. Sukhanov ${ }^{65}$, M. Suljic ${ }^{35}$, R. Sultanov ${ }^{95}$, M. Sumbera ${ }^{98}$, V. Sumberia ${ }^{104}$, S. Sumowidagdo ${ }^{52}$, S. Swain ${ }^{67}$, A. Szabo ${ }^{13}$, I. Szarka ${ }^{13}$, U. Tabassam ${ }^{14}$, S.F. Taghavi ${ }^{108}$, G. Taillepied ${ }^{137}$, J. Takahashi ${ }^{124}$, G.J. Tambave ${ }^{21}$, S. Tang ${ }^{137,7}$, Z. Tang ${ }^{131}$, J.D. Tapia Takaki ${ }^{\text {VII, }}{ }^{129}$, M. Tarhini ${ }^{117}$, M.G. Tarzila ${ }^{49}$, A. Tauro ${ }^{35}$, G. Tejeda Muñoz ${ }^{46}$, A. Telesca ${ }^{35}$, L. Terlizzi $^{25}$, C. Terrevoli ${ }^{127}$, G. Tersimonov ${ }^{3}$, S. Thakur ${ }^{143}$, D. Thomas ${ }^{121}$, R. Tieulent ${ }^{138}$, A. Tikhonov ${ }^{65}$, A.R. Timmins ${ }^{127}$,

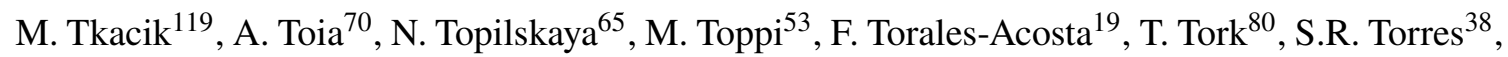
A. Trifiró ${ }^{33,57}$, S. Tripathy ${ }^{55,71}$, T. Tripathy ${ }^{50}$, S. Trogolo ${ }^{35,28}$, V. Trubnikov ${ }^{3}$, W.H. Trzaska ${ }^{128}$, T.P. Trzcinski ${ }^{144}$, B.A. Trzeciak $^{38}$, A. Tumkin ${ }^{111}$, R. Turrisi ${ }^{58}$, T.S. Tveter $^{20}$, K. Ullaland ${ }^{21}$, A. Uras ${ }^{138}$, M. Urioni ${ }^{59,142}$, G.L. Usai ${ }^{23}$, M. Vala ${ }^{39}$, N. Valle ${ }^{29,59}$, S. Vallero ${ }^{61}$, N. van der Kolk ${ }^{64}$, L.V.R. van Doremalen ${ }^{64}$, M. van Leeuwen ${ }^{93}$, P. Vande $V_{y v r e}{ }^{35}$, D. Varga ${ }^{147}$, Z. Varga ${ }^{147}$, M. Varga$\mathrm{Kofarago}^{147}$, A. Vargas ${ }^{46}$, M. Vasileiou ${ }^{87}$, A. Vasiliev ${ }^{91}$, O. Vázquez Doce ${ }^{53,108}$, V. Vechernin ${ }^{115}$, E. Vercellin ${ }^{25}$, S. Vergara Limón ${ }^{46}$, L. Vermunt ${ }^{64}$, R. Vértesi ${ }^{147}$, M. Verweij ${ }^{64}$, L. Vickovic ${ }^{36}$, Z. Vilakazi ${ }^{134}$, O. Villalobos Baillie ${ }^{113}$, G. Vino $^{54}$, A. Vinogradov ${ }^{91}$, T. Virgili ${ }^{30}$, V. Vislavicius ${ }^{92}$, A. Vodopyanov ${ }^{77}$, B. Volkel ${ }^{35}$, M.A. Völk1 ${ }^{107}$, K. Voloshin ${ }^{95}$, S.A. Voloshin ${ }^{145}$, G. Volpe ${ }^{34}$, B. von Haller $^{35}$, I. Vorobyev ${ }^{108}$, D. Voscek ${ }^{119}$, N. Vozniuk ${ }^{65}$, J. Vrláková ${ }^{39}$, B. Wagner ${ }^{21}$, C. Wang ${ }^{41}$, D. Wang ${ }^{41}$, M. Weber ${ }^{116}$, R.J.G.V. Weelden ${ }^{93}$, A. Wegrzynek ${ }^{35}$, S.C. Wenzel ${ }^{35}$, J.P. Wessels ${ }^{146}$, J. Wiechula ${ }^{70}$, J. Wikne ${ }^{20}$, G. Wilk ${ }^{88}$, J. Wilkinson ${ }^{110}$, G.A. Willems ${ }^{146}$, B. Windelband ${ }^{107}$, M. Winn ${ }^{140}$, W.E. Witt ${ }^{133}$, J.R. Wright ${ }^{121}$, W. Wu ${ }^{41}$, Y. Wu ${ }^{131}$, R. Xu ${ }^{7}$, A.K. Yadav ${ }^{143}$, S. Yalcin $^{79}$, Y. Yamaguchi ${ }^{47}$, K. Yamakawa ${ }^{47}$, S. Yang ${ }^{21}$, S. Yano ${ }^{47}$, Z. Yin ${ }^{7}$, H. Yokoyama ${ }^{64}$, I.-K. Yoo ${ }^{17}$, J.H. Yoon ${ }^{63}$, S. Yuan ${ }^{21}$, A. Yuncu ${ }^{107}$, V. Zaccolo ${ }^{24}$, C. Zampolli ${ }^{35}$, H.J.C. Zanoli ${ }^{64}$, N. Zardoshti ${ }^{35}$, A. Zarochentsev ${ }^{115}$, P. Závada ${ }^{68}$, N. Zaviyalov ${ }^{111}$, M. Zhalov ${ }^{101}$, B. Zhang ${ }^{7}$, S. Zhang ${ }^{41}$, X. Zhang ${ }^{7}$, Y. Zhang ${ }^{131}$, V. Zherebchevskii ${ }^{115}$, Y. Zhi ${ }^{11}$, N. Zhigareva ${ }^{95}$, D. Zhou ${ }^{7}$, Y. Zhou ${ }^{92}$, J. Zhu ${ }^{7}{ }^{110}$, Y. Zhu ${ }^{7}$, A. Zichichi ${ }^{26}$, G. Zinovjev ${ }^{3}$, N. Zurlo ${ }^{142,59}$

\section{Affiliation Notes}

\section{Deceased}

II Also at: Italian National Agency for New Technologies, Energy and Sustainable Economic Development (ENEA), Bologna, Italy

III Also at: Dipartimento DET del Politecnico di Torino, Turin, Italy

IV Also at: M.V. Lomonosov Moscow State University, D.V. Skobeltsyn Institute of Nuclear, Physics, Moscow, Russia 
V Also at: Department of Applied Physics, Aligarh Muslim University, Aligarh, India

VI Also at: Institute of Theoretical Physics, University of Wroclaw, Poland

VII Also at: University of Kansas, Lawrence, Kansas, United States

\section{Collaboration Institutes}

${ }^{1}$ A.I. Alikhanyan National Science Laboratory (Yerevan Physics Institute) Foundation, Yerevan, Armenia

${ }^{2}$ AGH University of Science and Technology, Cracow, Poland

${ }^{3}$ Bogolyubov Institute for Theoretical Physics, National Academy of Sciences of Ukraine, Kiev, Ukraine

${ }^{4}$ Bose Institute, Department of Physics and Centre for Astroparticle Physics and Space Science (CAPSS), Kolkata, India

${ }^{5}$ Budker Institute for Nuclear Physics, Novosibirsk, Russia

${ }^{6}$ California Polytechnic State University, San Luis Obispo, California, United States

${ }^{7}$ Central China Normal University, Wuhan, China

${ }^{8}$ Centro de Aplicaciones Tecnológicas y Desarrollo Nuclear (CEADEN), Havana, Cuba

${ }^{9}$ Centro de Investigación y de Estudios Avanzados (CINVESTAV), Mexico City and Mérida, Mexico

${ }^{10}$ Chicago State University, Chicago, Illinois, United States

${ }^{11}$ China Institute of Atomic Energy, Beijing, China

${ }^{12}$ Chungbuk National University, Cheongju, Republic of Korea

${ }^{13}$ Comenius University Bratislava, Faculty of Mathematics, Physics and Informatics, Bratislava,

Slovakia

${ }^{14}$ COMSATS University Islamabad, Islamabad, Pakistan

${ }^{15}$ Creighton University, Omaha, Nebraska, United States

${ }^{16}$ Department of Physics, Aligarh Muslim University, Aligarh, India

${ }^{17}$ Department of Physics, Pusan National University, Pusan, Republic of Korea

${ }^{18}$ Department of Physics, Sejong University, Seoul, Republic of Korea

${ }^{19}$ Department of Physics, University of California, Berkeley, California, United States

${ }^{20}$ Department of Physics, University of Oslo, Oslo, Norway

${ }^{21}$ Department of Physics and Technology, University of Bergen, Bergen, Norway

${ }^{22}$ Dipartimento di Fisica dell'Università 'La Sapienza' and Sezione INFN, Rome, Italy

${ }^{23}$ Dipartimento di Fisica dell'Università and Sezione INFN, Cagliari, Italy

${ }^{24}$ Dipartimento di Fisica dell'Università and Sezione INFN, Trieste, Italy

${ }^{25}$ Dipartimento di Fisica dell'Università and Sezione INFN, Turin, Italy

${ }^{26}$ Dipartimento di Fisica e Astronomia dell'Università and Sezione INFN, Bologna, Italy

${ }^{27}$ Dipartimento di Fisica e Astronomia dell'Università and Sezione INFN, Catania, Italy

${ }^{28}$ Dipartimento di Fisica e Astronomia dell'Università and Sezione INFN, Padova, Italy

${ }^{29}$ Dipartimento di Fisica e Nucleare e Teorica, Università di Pavia, Pavia, Italy

${ }^{30}$ Dipartimento di Fisica 'E.R. Caianiello' dell'Università and Gruppo Collegato INFN, Salerno, Italy 
${ }^{31}$ Dipartimento DISAT del Politecnico and Sezione INFN, Turin, Italy

32 Dipartimento di Scienze e Innovazione Tecnologica dell'Università del Piemonte Orientale and INFN Sezione di Torino, Alessandria, Italy

${ }^{33}$ Dipartimento di Scienze MIFT, Università di Messina, Messina, Italy

${ }^{34}$ Dipartimento Interateneo di Fisica 'M. Merlin' and Sezione INFN, Bari, Italy

${ }^{35}$ European Organization for Nuclear Research (CERN), Geneva, Switzerland

${ }^{36}$ Faculty of Electrical Engineering, Mechanical Engineering and Naval Architecture, University of Split, Split, Croatia

${ }^{37}$ Faculty of Engineering and Science, Western Norway University of Applied Sciences, Bergen, Norway

${ }^{38}$ Faculty of Nuclear Sciences and Physical Engineering, Czech Technical University in Prague, Prague, Czech Republic

${ }^{39}$ Faculty of Science, P.J. Šafárik University, Košice, Slovakia

${ }^{40}$ Frankfurt Institute for Advanced Studies, Johann Wolfgang Goethe-Universität Frankfurt, Frankfurt, Germany

${ }^{41}$ Fudan University, Shanghai, China

${ }^{42}$ Gangneung-Wonju National University, Gangneung, Republic of Korea

${ }^{43}$ Gauhati University, Department of Physics, Guwahati, India

${ }^{44}$ Helmholtz-Institut für Strahlen- und Kernphysik, Rheinische Friedrich-Wilhelms-Universität Bonn, Bonn, Germany

${ }^{45}$ Helsinki Institute of Physics (HIP), Helsinki, Finland

${ }^{46}$ High Energy Physics Group, Universidad Autónoma de Puebla, Puebla, Mexico

${ }^{47}$ Hiroshima University, Hiroshima, Japan

${ }^{48}$ Hochschule Worms, Zentrum für Technologietransfer und Telekommunikation (ZTT), Worms, Germany

${ }^{49}$ Horia Hulubei National Institute of Physics and Nuclear Engineering, Bucharest, Romania

${ }^{50}$ Indian Institute of Technology Bombay (IIT), Mumbai, India

${ }^{51}$ Indian Institute of Technology Indore, Indore, India

52 Indonesian Institute of Sciences, Jakarta, Indonesia

${ }^{53}$ INFN, Laboratori Nazionali di Frascati, Frascati, Italy

54 INFN, Sezione di Bari, Bari, Italy

${ }^{55}$ INFN, Sezione di Bologna, Bologna, Italy

${ }^{56}$ INFN, Sezione di Cagliari, Cagliari, Italy

${ }^{57}$ INFN, Sezione di Catania, Catania, Italy

58 INFN, Sezione di Padova, Padova, Italy

${ }^{59}$ INFN, Sezione di Pavia, Pavia, Italy

${ }^{60}$ INFN, Sezione di Roma, Rome, Italy

${ }^{61}$ INFN, Sezione di Torino, Turin, Italy

62 INFN, Sezione di Trieste, Trieste, Italy

${ }^{63}$ Inha University, Incheon, Republic of Korea

${ }^{64}$ Institute for Gravitational and Subatomic Physics (GRASP), Utrecht University/Nikhef, Utrecht, Netherlands

${ }^{65}$ Institute for Nuclear Research, Academy of Sciences, Moscow, Russia 
${ }^{66}$ Institute of Experimental Physics, Slovak Academy of Sciences, Košice, Slovakia

${ }^{67}$ Institute of Physics, Homi Bhabha National Institute, Bhubaneswar, India

${ }^{68}$ Institute of Physics of the Czech Academy of Sciences, Prague, Czech Republic

${ }^{69}$ Institute of Space Science (ISS), Bucharest, Romania

${ }^{70}$ Institut für Kernphysik, Johann Wolfgang Goethe-Universität Frankfurt, Frankfurt, Germany

${ }^{71}$ Instituto de Ciencias Nucleares, Universidad Nacional Autónoma de México, Mexico City, Mexico

72 Instituto de Física, Universidade Federal do Rio Grande do Sul (UFRGS), Porto Alegre, Brazil

${ }^{73}$ Instituto de Física, Universidad Nacional Autónoma de México, Mexico City, Mexico

74 iThemba LABS, National Research Foundation, Somerset West, South Africa

75 Jeonbuk National University, Jeonju, Republic of Korea

76 Johann-Wolfgang-Goethe Universität Frankfurt Institut für Informatik, Fachbereich Informatik und Mathematik, Frankfurt, Germany

77 Joint Institute for Nuclear Research (JINR), Dubna, Russia

${ }^{78}$ Korea Institute of Science and Technology Information, Daejeon, Republic of Korea

${ }^{79}$ KTO Karatay University, Konya, Turkey

${ }^{80}$ Laboratoire de Physique des 2 Infinis, Irène Joliot-Curie, Orsay, France

${ }^{81}$ Laboratoire de Physique Subatomique et de Cosmologie, Université Grenoble-Alpes, CNRS-

IN2P3, Grenoble, France

${ }^{82}$ Lawrence Berkeley National Laboratory, Berkeley, California, United States

${ }^{83}$ Lund University Department of Physics, Division of Particle Physics, Lund, Sweden

${ }^{84}$ Moscow Institute for Physics and Technology, Moscow, Russia

${ }^{85}$ Nagasaki Institute of Applied Science, Nagasaki, Japan

${ }^{86}$ Nara Women's University (NWU), Nara, Japan

${ }^{87}$ National and Kapodistrian University of Athens, School of Science, Department of Physics , Athens, Greece

${ }^{88}$ National Centre for Nuclear Research, Warsaw, Poland

${ }^{89}$ National Institute of Science Education and Research, Homi Bhabha National Institute, Jatni, India

${ }^{90}$ National Nuclear Research Center, Baku, Azerbaijan

${ }^{91}$ National Research Centre Kurchatov Institute, Moscow, Russia

92 Niels Bohr Institute, University of Copenhagen, Copenhagen, Denmark

${ }^{93}$ Nikhef, National institute for subatomic physics, Amsterdam, Netherlands

${ }^{94}$ NRC Kurchatov Institute IHEP, Protvino, Russia

${ }^{95} \mathrm{NRC}$ «Kurchatov»Institute - ITEP, Moscow, Russia

${ }^{96}$ NRNU Moscow Engineering Physics Institute, Moscow, Russia

${ }^{97}$ Nuclear Physics Group, STFC Daresbury Laboratory, Daresbury, United Kingdom

${ }^{98}$ Nuclear Physics Institute of the Czech Academy of Sciences, Řež u Prahy, Czech Republic

99 Oak Ridge National Laboratory, Oak Ridge, Tennessee, United States

100 Ohio State University, Columbus, Ohio, United States

${ }^{101}$ Petersburg Nuclear Physics Institute, Gatchina, Russia

102 Physics department, Faculty of science, University of Zagreb, Zagreb, Croatia

${ }^{103}$ Physics Department, Panjab University, Chandigarh, India 
${ }^{104}$ Physics Department, University of Jammu, Jammu, India

105 Physics Department, University of Rajasthan, Jaipur, India

106 Physikalisches Institut, Eberhard-Karls-Universität Tübingen, Tübingen, Germany

${ }^{107}$ Physikalisches Institut, Ruprecht-Karls-Universität Heidelberg, Heidelberg, Germany

108 Physik Department, Technische Universität München, Munich, Germany

109 Politecnico di Bari and Sezione INFN, Bari, Italy

${ }^{110}$ Research Division and ExtreMe Matter Institute EMMI, GSI Helmholtzzentrum für Schwerionenforschung GmbH, Darmstadt, Germany

${ }^{111}$ Russian Federal Nuclear Center (VNIIEF), Sarov, Russia

${ }^{112}$ Saha Institute of Nuclear Physics, Homi Bhabha National Institute, Kolkata, India

${ }^{113}$ School of Physics and Astronomy, University of Birmingham, Birmingham, United Kingdom

${ }^{114}$ Sección Física, Departamento de Ciencias, Pontificia Universidad Católica del Perú, Lima, Peru

115 St. Petersburg State University, St. Petersburg, Russia

${ }^{116}$ Stefan Meyer Institut für Subatomare Physik (SMI), Vienna, Austria

117 SUBATECH, IMT Atlantique, Université de Nantes, CNRS-IN2P3, Nantes, France

118 Suranaree University of Technology, Nakhon Ratchasima, Thailand

119 Technical University of Košice, Košice, Slovakia

120 The Henryk Niewodniczanski Institute of Nuclear Physics, Polish Academy of Sciences, Cracow, Poland

121 The University of Texas at Austin, Austin, Texas, United States

${ }^{122}$ Universidad Autónoma de Sinaloa, Culiacán, Mexico

${ }^{123}$ Universidade de São Paulo (USP), São Paulo, Brazil

124 Universidade Estadual de Campinas (UNICAMP), Campinas, Brazil

125 Universidade Federal do ABC, Santo Andre, Brazil

126 University of Cape Town, Cape Town, South Africa

${ }^{127}$ University of Houston, Houston, Texas, United States

128 University of Jyväskylä, Jyväskylä, Finland

${ }^{129}$ University of Kansas, Lawrence, Kansas, United States

${ }^{130}$ University of Liverpool, Liverpool, United Kingdom

${ }^{131}$ University of Science and Technology of China, Hefei, China

132 University of South-Eastern Norway, Tonsberg, Norway

${ }^{133}$ University of Tennessee, Knoxville, Tennessee, United States

${ }^{134}$ University of the Witwatersrand, Johannesburg, South Africa

${ }^{135}$ University of Tokyo, Tokyo, Japan

136 University of Tsukuba, Tsukuba, Japan

137 Université Clermont Auvergne, CNRS/IN2P3, LPC, Clermont-Ferrand, France

138 Université de Lyon, CNRS/IN2P3, Institut de Physique des 2 Infinis de Lyon, Lyon, France

${ }^{139}$ Université de Strasbourg, CNRS, IPHC UMR 7178, F-67000 Strasbourg, France, Strasbourg,

France

${ }^{140}$ Université Paris-Saclay Centre d'Etudes de Saclay (CEA), IRFU, Départment de Physique Nucléaire $(\mathrm{DPhN})$, Saclay, France

${ }^{141}$ Università degli Studi di Foggia, Foggia, Italy

142 Università di Brescia, Brescia, Italy 
143 Variable Energy Cyclotron Centre, Homi Bhabha National Institute, Kolkata, India

${ }^{144}$ Warsaw University of Technology, Warsaw, Poland

${ }^{145}$ Wayne State University, Detroit, Michigan, United States

146 Westfälische Wilhelms-Universität Münster, Institut für Kernphysik, Münster, Germany

147 Wigner Research Centre for Physics, Budapest, Hungary

148 Yale University, New Haven, Connecticut, United States

149 Yonsei University, Seoul, Republic of Korea 